\title{
Adaptation of a ClassRoom ObSERVATION Protocol FOR ACTIVE LEARNING
}

\author{
Allison Van Beek and Susan McCahan \\ University of Toronto \\ allison.vanbeek@utoronto.ca
}

\begin{abstract}
In our work, we are focusing on the use of classroom observation to provide feedback on instructional space design. An initiative to redesign teaching space began a decade ago at a large, research-intensive institution. In September 2018, a large-scale (477 seat) active learning classroom became operational. The affordances of this space are intended to enable active teaching and learning in large classes. However, it is difficult to assess how successful this space is for active learning. A multi-year study has been undertaken to observe teaching practice in situ, with the goal of developing design principles for instructional space and technology that support the development, design, and implementation of teaching activities.

Existing teaching observation protocols do not fully capture the interaction between the instructor and the space because such protocols were generally intended for other purposes. The goal is to develop a protocol that captures activities that are both intrinsic and extrinsic to teaching.

This paper describes the development and use of an observation protocol. The core of the protocol is the wellknown Teaching Dimensions Observation Tool (TDOP). The scope of the TDOP is extended to active learning activities drawing from the Active Learning Classroom Observation Tool (ALCOT). The resulting extended protocol, TDOP+, was used for coding both live and recorded classroom observations in the Winter 2020 term. This extended protocol allows the researchers to capture information that characterizes the intersection of pedagogy, space, and technology through Activity Theory. In future work, the data gathered through observations will be analyzed using the Differentiated Overt Learning Activities (DOLA) framework, to provide insight into what types of teaching activity happens in a large-scale active learning classroom across STEM education and how active learning in large classrooms compares to pedagogy in other spaces.
\end{abstract}

Keywords: Active Learning, Classroom Observation Protocol, Learning environment, Learning technology, STEM

\section{INTRODUCTION}

Classroom observations have been used for a multitude of purposes from providing feedback to instructors to informing program design and exploring how widely a pedagogy has been implemented [26]. In our work, we are focusing on a different use of a classroom observation protocol: the use of classroom observation to provide feedback on instructional space design. An initiative to redesign teaching space began a decade ago at a large research-intensive institution. This initiative, like work happening at other institutions, has its roots in research on active teaching dating back to the 1970s [1], [29], [8], [28]. In September 2018, a large-scale (477 seat) active learning classroom in engineering became operational. The affordances of this space are intended to enable large-scale active teaching and learning. However, it is difficult to assess how successful the design of spaces like this are for active learning [22]. Active learning practices are not being incorporated as widely as expected in some STEM fields [14] and other active learning classroom installations report an increased volume of support requests related to the transition from traditional to active learning classrooms [2].

We are undertaking a multi-year study to observe teaching practice in situ, with the goal of developing design principles for instructional space and technology to support active learning and mitigate the need for excessive technical support. The ultimate goal is to build facilities that support the development, design, and implementation of teaching activities particularly for large-scale active learning.

While students can learn from passive pedagogies, they learn more from active pedagogies [4]. In 2009, Chi developed the Differentiated Overt Learning Activity (DOLA) framework, which uses the Interactive, Constructivist, Active, Passive (ICAP) hypothesis, to subdivides the descriptor "active learning" into more specific activities [16]. This framework is predicated on the idea that it is difficult to detect the differences in types of active learning, especially between students triggering knowledge (being active), building their own knowledge (being constructive), and engaging with others in activities that grow knowledge (being interactive) [16]. Because we are studying a large-scale active learning classroom -- a space purposebuilt to support active learning -- we want to observe activity using a method that would allow for comparison across courses, teaching teams, and academic terms using the DOLA framework.

An ideal observation protocol for this project should capture information on both intrinsic and extrinsic teaching activities. Intrinsic teaching activities include traditional lecturing, and the time spent preparing the learners to participate in an activity, time learners spend on an activity, 
and review or reflection. Observation protocols often focus on intrinsic activities. Extrinsic elements, in contrast, are activities that do not move the course forward per se [7]. They can be administrative, organizational, or emergent. Extrinsic elements include technology outages, review of previously taught material, and administrative activities such as handing back papers. In our work, extrinsic activities are critical to understanding how the space and technology support, or fail to support, the instructor's intended class activities. Activity Theory, the theoretical underpinning of the research, suggests taking a holistic view of the active learning ecosystem, achieved through understanding the goals of the people acting in and through the space [19], [11], [13], [24], [21] which includes both intrinsic and extrinsic activities.

In brief, we are looking for a validated classroom observation protocol that provides information relevant for space and technology design. Ideally, such a protocol aligns with an Activity Theory framework as well as commonly implemented active learning techniques in engineering. In addition to alignment with theory, we are also seeking a protocol that is:

1. Descriptive - many protocols are evaluative, providing comment on the success of pedagogical implementation, which is not the intent of this research project.

2. Pedagogically agnostic - coding for a broad spectrum of intrinsic and extrinsic teaching activity versus looking for the implementation of a specific pedagogy.

3. Holistic - coding for each element of the active learning ecosystem as well as their relationship to each other.

4. Designed for use within STEM disciplines - while teaching activity could be common across domains, seeking a protocol that already encompasses frequently used activities in STEM fields is beneficial to the project because we are focusing on engineering active learning spaces.

These attributes help to ensure an alignment with Activity Theory by enabling the observer to document teaching activity in an active learning classroom in a way that captures its complex and dynamic nature [16]. We aim to holistically describe teaching as a complex, dynamic system in order to understand STEM active learning practice. The goal is to document interactions between actors and artifacts (e.g. technology, furniture, etc.) to see how (and if) classroom norms, the divisions of labour, and the uses of classroom technology change over time [5].

Based on a review of existing classroom observation protocols [28], we determined that there is currently no protocol that is a good fit for our study. In this paper, we describe the process of developing the extended Teaching Dimensions Observation Protocol (TDOP+) and what we learned about the new extended protocol during its pilot use in the Winter 2020 academic term.

CEEA-ACEG21; Paper 142

University of Prince Edward Island; June 21 - 23, 2021- 2 of 9 -

\section{INTRODUCTION TO THE OBSERVATION PRotocols}

In our review of existing protocols [27], two emerged as potentially well suited to this project - the Active Learning Classroom Observation Tool (ALCOT) [2] and the Teaching Dimensions Observation Protocol (TDOP) [16]. In 2016, Birdwell et al. [2] suggested that there are no existing observation protocols or tools that emphasize the active learning ecosystems per se - the intersection of space, technology, and pedagogy - as important considerations that factor into the context of teaching. As a result, they proposed their own: The ALCOT [2]. The ALCOT aligns with two of the four attributes we identified: it is a holistic protocol, and it is pedagogically agnostic. It is designed to provide feedback to instructors on their use of active learning spaces, specifically including space and technology affordances, in a variety of rooms with varying instructional needs.

The result is a protocol composed of four categories:

1. support of active learning,

2. creation and implementation of student collaborative learning activities,

3. formative assessment in the classroom, and

4. classroom management.

The ALCOT is a three-stage observation tool, including a Pre-Observation Checklist, a chronological note-taking instrument, and post-observation feedback via an Active Learning Classroom Observation Tool. The ALCOT is open-ended; observations are recorded qualitatively. While this type of developmental feedback is likely invaluable when provided to an individual instructor, the protocol does not offer the macro-view of the teaching activity across courses that is desired for our work. However, it is the only widely used protocol that specifically captures active learning strategy implementation, including the use of space and technology [2].

The TDOP, in contrast to the ALCOT, is a customizable observation protocol designed to quantitatively capture the dynamics of teaching and learning through the coding of observed behaviours [17]. It meets each of the attributes identified for this project: it is a descriptive protocol, it is pedagogically agnostic, it is holistic, and it was designed for use in the STEM domain (although the designers encourage expansion to all academic domains) [17]. However, it lacks specific observational behaviours known to be present in active learning.

The TDOP captures the multi-dimensional nature of classroom activities, reflecting a complex environment consisting of multiple actors and artifacts by coding observable concrete activity in educational environments at a granular level [16]. The focus on task performance, classroom norms, and the level of granularity of data collection assists in revealing the underlying nature of the different types of pedagogical activity being employed. By paying close 
attention to how tools and artifacts mediate activity over time, it is intended to capture how teaching practice evolves without including value judgements regarding the effectiveness of instructional practice or commenting on successful alignment with a specific pedagogy [25].

The TDOP is divided into five aspects of classroom dynamics:

1. teaching methods,

2. pedagogical strategies,

3. types of student-instructor interactions,

4. types of cognitive engagement, and

5. use of instructional technology.

Each category has a set of codes to track observable behaviours. These codes can be combined to represent pedagogical constructs, if and as needed [16]; e.g., an active learning activity, which is composed of multiple segments. For example, to capture an activity in full, the behavioural pattern on the timeline could include the time spent on preparing learners to participate in an activity, performing any organizational tasks, completing the activity, and any review or summary time. This combination of codes - this pedagogical construct - would be considered an "active learning activity" and could tell a robust story about the nature and timing of the learning activity. In future analysis, we plan to use the ICAP Framework (i.e. DOLA), to further contextualize the data and to allow comparison of active learning implementation patterns in this particular active learning space versus other active learning spaces. Previous observations using the TDOP have been analyzed using this framework [16].

By segmenting complex human action into smaller tasks, it becomes possible to see how different operations, actions, and activities occur in relation to each other and how (or if) these relationships change over time as well as if tensions between elements arise. It is also possible to see if activities are carried out by different actors in the learning space, if they occur with different frequency, or if they are likely to occur in combination with other activities. The TDOP fulfills many of the requirements for the protocol needed for this research and it is a protocol that has already been validated and implemented widely. However, Hora makes the point that the TDOP should be customized to fit the different foci of each research project [15].

\section{Modifying the Protocols}

The TDOP and the ALCOT each has attributes that we need for our project. To build the TDOP+, we extended the base codes used in the TDOP with operationalized active learning activities included in the ALCOT. A full list of the codes is given in Appendix A which shows the original TDOP framework, and the new codes that we have added. By building on complementary aspects of TDOP and ALCOT, the TDOP + could meet all the identified required attributes.
These modifications fall into three main categories:

1. Active Learning Components - converting the inferential elements of ALCOT that rely on judgements by the observer into concrete, observable behaviours with associated codes.

2. Extrinsic Activities - adding codes to provide depth and description about how time is spent during class time, particularly for activities that are administrative in nature.

3. Tools - adding codes that account for the unique and specific tool/technology affordances of the room.

The ALCOT captures important active learning instructional strategies not captured in the TDOP, but the ALCOT elicits subjective judgements. To extract the value of the ALCOT framework for our project, we converted its inferential elements into observable behaviours that can be coded. An example of this is the ALCOT question, "How did the instructor provide feedback to students during activities or assessments?" [2]. The TDOP includes comprehensive codes for interactions (e.g., different types of questions to learners; classroom response tools (CRTs)) and based on the ALCOT we added two code: Preparation (P) and Review (RE). This allows us to capture when time is spent pre-activity preparing students for the activity as well as post-activity summarizing and debriefing with the class. Codes are stackable; behaviours can be concurrently coded (e.g., Review also includes use of a CRT). This is called "co-coding".

To merge these protocols, we first expanded existing child attributes within a parent TDOP categories if possible, and then added new sub-dimensions if necessary. In the spirit of the TDOP, the additions are concrete, observable behaviours that add to the description of the active learning ecosystem.

Adding codes required trade-offs, as each behaviour added to the protocol makes the task of observation more difficult. Yet without modification, it does not fully capture the information needed to support classroom space and technology design. We noticed that the TDOP protocol captures intrinsic activity better than extrinsic activity. A further goal is to capture collaborative and team-teaching activity which is a category of activities missing from both the TDOP and ALCOT protocols.

Developing a practicable and reliable protocol that captures the activity in the room involved testing. In this paper, we are describing preliminary work. While the translation of the ALCOT to observable codes in the TDOP was completed prior to the start of observations, identifying the full scope of extrinsic activities and tools that require codes was resolved only after testing the protocol in situ. When we observed an activity or tool that was not encompassed by the protocol, it was noted. The ethogram was then extended further by adding a code for that activity. 


\subsection{Examples of ALCOT-based Modifications}

Here we describe examples of codes that were added to the TDOP based on the ALCOT prior to in-class observations.

Example \#1: In the ALCOT, the observer is asked to answer the question, "How did the instructor provide directions for collaborative activities?" [2]. To capture this intent, we added codes for Preparation/Planning to the Teaching Methods dimension in TDOP. The Preparation/Planning $(\mathrm{P})$ code is used if time is explicitly spent explaining the context of an activity, setting up a tool for use during the activity, or configuring a demonstration for learners.

Example \#2: The Review/Recapitulation (RE) code was added to capture the time spent after an active learning activity is completed. This is the complementary code to the Preparation code and characterizes how the instructor wraps up an active learning activity. It tracks whether time is spent reviewing or summarizing student activity. The RE code allows tracking of the verbal (or digitally displayed) debrief portion of an active learning activity and is often co-coded with technology codes (e.g. CRTs) and assessment codes.

These codes (i.e., $\mathrm{P}$ and RE) were added prior to the start of the observations by identifying how the TDOP could be extended using the ALCOT. The addition of these intrinsic teaching codes helps to accurately record the time spent on active teaching and segments the activity in a way that becomes useful for determining patterns and time efficiency.

\section{PRELIMINARY OBSERVATIONS}

The TDOP+ was used to observe 10 STEM courses through the first half of the Winter 2020 term (REB approved: 00038186). All instructors (19) teaching in the space were invited to participate; 12 participated, 1 declined and 6 did not respond. There were 8 courses from the Faculty of Applied Science and Engineering and 2 from the Faculty of Arts and Science; all were STEM courses. Most of the participants were teaching first year courses. Solo, pair, and team-teaching structures were represented in the sample. Due to the COVID-19 shutdown, the inperson observations were suspended about two-thirds of the way through the term. The plan was to capture $33 \%$ of all class sessions; instead about $20 \%$ of each course was observed.

This breadth of courses allowed us to observe a variety of different pedagogical styles and teaching teams in action. Capturing the different styles of courses using the new protocol was challenging. To facilitate coding, we used BORIS (Behavioral Observation Research Interactive Software), which is an open-source observation tool that allows the investigator to build their own ethogram to facilitate live coding. The TDOP normally codes in twominute time increments, but BORIS allows the observer to track activity by duration more precisely. This produces a more detailed timeline. While learning to use BORIS took only a few hours of practice and training, capturing the span of activity occurring in an active learning classroom required the full semester to refine. In addition, based on our preliminary observations, we added extrinsic activity codes to the TDOP.

The following examples illustrate the types of extrinsic activities we observed. Extrinsic activity (e.g., time spent launching a video) may, or may not, be built into the instructor's teaching plan. However, extrinsic activity may take longer than anticipated and the time spent is unavoidable and often frustrating for instructors [7]. The TDOP does not natively code for this type of activity. During preliminary testing of the protocol, it was evident that these extrinsic elements make up a significant portion of some classes and often involved tool set up and collaborative teaching.

Example \#3: We added the code Regrouping (RG) to describe when the instructor takes time to reflect or to prepare themselves for the next activity. It requires a temporary change of focus from instructing or facilitating to contemplating their next move, either individually or with teaching team members. This is different from the Preparation (P) code, described earlier, which is a studentcentered code used when the instructor is explaining the activity for the students. Regrouping (RG) is an instructorcentered code, used when the instructor needs to take time for themselves to recalibrate next steps like reorganizing the lesson plan or reordering activities. It is intended to depict when there is a pause, pivot, or time taken to decide on next steps in the class session orchestration. This code was added when it was observed that teaching team members almost universally took time to themselves regularly as part of their class; it is often done at the beginning of a student-centered activity and it decreased the amount of time for active instruction.

Example \#4: The Collaborative/Complementary Teaching (CT) code was added to the Pedagogical Strategies section to capture team teaching practices. This code allows the observer to track when there is more than one instructor as the focal point during the class session, and could include others who might take on this role (e.g., teaching assistant, guest lecturer, and so on). Increasingly, active learning is not managed by an individual instructor but by a teaching team and this collaboration can manifest in regrouping, check-ins, and activities that take up time during the class. Team roles and their division of labour is unique to each class, not only because it includes a mix of instructors, teaching assistants, technical support staff, and others, but also because each individual actor in the space brings their own experiences and histories.

\subsection{Classroom Technology}

As we stated in the introduction, the purpose of this study is to inform space and technology design in large 
active learning classrooms. From an Activity Theory perspective, the interaction between the people in the space (students and instructors) and the mediating, contextspecific technology is critical to this purpose. For this reason, in addition to expanding the TDOP based on the ALCOT and observations, we also expanded the existing list of Instructional Technology codes to identify the tools specific to the space we are studying, as Hora [16] encourages users of the TDOP to do. There are instances where neither TDOP nor ALCOT effectively capture the new technology-enabled learning ecosystem that exists in modern active learning classrooms. This expansion includes tracking technology use such as the use of a timer, Wi-Fi display connection, HDMI table inputs, audience (student) microphones, cameras, a document camera, hard copy classroom response tools (e.g., colour-coded cards), and other technologies. Basically, we would like to capture information about all technology use in the instructional ecosystem and situate this information in the class session timeline. Each code has the option of attributes; for the instructional tool code, we added the ability to track tool use success or failure.

Example \#5: Moving from traditional classrooms to active learning classrooms requires a recontextualization of teaching and learning environments rather than a direct translation of educational practices in a new setting [12]. If technical support was requested during a class, it was coded under another new code, technical assistance (TECH). One of our goals is to provide feedback on the design of the classroom. By providing insight into where the technology is failing, or when frustration is expressed, patterns may emerge that demonstrate when an activity is successfully enabled or if something (e.g., support or reliability) is lacking. Currently, there is no coding for other physical limitations in the room (e.g., screen visibility, lack of outlets) but this is something that could be considered in future protocol iterations.

\section{Discussion: The Protocol in USE}

The protocol was used for coding both live and recorded class sessions that occurred in the Winter 2020 term. The TDOP+ was used for both types of observations. In our preliminary reflections on this process, we note that video observations result in about three times the number of codes because the observer-controlled playback of the video allowed for a significantly greater number of activity elements to be coded than was possible in real-time. Coding using the video recordings, however, is significantly more time consuming; one 50 -minute class requires approximately 3 hours of coding.

Consistency between in-person and video observations is an ongoing challenge. Classroom observation protocols rely on the observer to infer whether a specific behaviour is occurring [16]. For example, the TDOP includes several different lecture codes - lecturing,
Socratic lecturing, lecturing while writing, lecturing while demonstrating, and each style can be co-coded with tool use. Additionally, you might have another element, like working out problems. Add in physical movement (e.g., instructors are frequently moving in the space, which is reflected in physical location codes) and the intricacies of the activity can result in an observation with some variance or even missing activity.

We address this challenge not by simplifying the complexity of the activity when translating it into codes, but by comparing the in-person and video coding to ensure that as much of the activity is coded as precisely as possible. It has been noted that a lack of inter-rate reliability is less problematic with descriptive protocols versus evaluative protocols [16]. It has been reassuring to observe that the overall patterns of activity within the class, as captured in the coded timeline, are similar via both video and in-person coding. Preliminary data analysis suggests differences in the volume of codes, but the general timeline of the class - its overall orchestration -- is essentially consistent. In the future we will have another observer independently check the coding.

The TDOP+ was designed to capture the unique and complex timeline of each class and the interactions that constitute formalized teaching in an instructional space, which is an intersection missing from existing classroom observation tools [2]. The TDOP+ has the potential to capture and describe the activity in the classroom, to inform design recommendations for future active learning classroom designs as well as to provide transparency into the type of teaching and learning activity that happens in this classroom. It also has the potential to provide valuable insight regarding how active learning classes are orchestrated and addresses the challenge of providing transparency into what types of teaching strategies are manifested in active learning classrooms, an area that currently lacks documentation.

The TDOP + is not designed to evaluate the success of these pedagogical strategies nor measure or comment on student performance. As a descriptive instrument, this is one of the limitations of the protocol and the resulting data. The TDOP+ does not provide insight into whether any particular pedagogy is being implemented effectively or strategically [16]. However, in conjunction with instructor interviews, the results of our coding could be used to suggest approaches to class orchestration that may support instructor goals more effectively. The timelines and other data (e.g., teaching team interaction information) may also prove to be a valuable focal point for instructor reflection.

\section{CONCLUSION}

The TDOP + is a first step toward achieving our research goals: 1) to develop a protocol that can be used to gather information to inform the design of future active learning classrooms and 2) to provide insight into what 
types of active learning activities are happening across STEM courses in a large-scale active learning classroom. Detailed coding and analysis of instructor activity is valuable information that can support the design process by providing detailed statistics on use and usability. Unlike other protocols, the TDOP+ was developed to provide descriptive feedback which can also inform changes or modification to teaching strategy. The TDOP was intended to be modified; and it is expected that investigators using the protocol will modify it for their own purposes, capturing the uniqueness of their specific active learning environments [17].

While the TDOP+ was designed for this specific research project, it could be used in other active learning spaces. It would be interesting to see how and if patterns of activity differ between different types of active learning classrooms and instructors. Further value can be added through the use of BORIS, which enables researchers to customize their observation protocol via the BORIS interface, capturing observation attributes as needed by the project. This customizability encourages its use within many different types of spaces, including traditional classrooms and non-formal teaching and learning spaces.

Data generated using the TDOP + protocol can be analyzed using the DOLA framework to provide comparable and concrete information about what teaching techniques (interactive, constructive, active, and passive) are currently used in Engineering active learning courses. This information will contribute to our understanding of active STEM education. By maintaining the integrity of the existing TDOP teaching dimensions, which is a validated protocol, we have ensured that the data collected using the modified protocol are comparable to other projects using the TDOP (or their own versions of the TDOP). A promising use for the merged observation protocol is its value to support professional development because it provides detailed feedback without being evaluative [16]. The data will also provide insight to instructors about any discrepancies between their perception of how time is spent in their class versus how time is actually spent. However, it is worth noting that although the TDOP+ has potential for widespread application, the data collected on any one space, by one observer, has limitations in generalizability.

The effects of the COVID-19 disruption are difficult to predict at this point in time and our future efforts will depend on being able to continue this research in a physical classroom. We hope to complete a second round of observations using the TDOP+. We believe, after completing both in-person and video observations using the new protocol, that the protocol has reached stability for use in this project. Ideas for future research include comparing the pre-COVID dataset we collected in Winter 2020 with a post-COVID dataset; with the thought that there could be a general upskilling in the technical proficiency of instructors or a change to teaching strategies. Future research areas could include whether the data reflect a shift in how classes are structured (generally or by individual instructors). We also expect that by using an established active learning activity taxonomy to analyze teaching in this and other classrooms, we will be able to inform the design of future classrooms.

\section{Acknowledgements}

All procedures performed in studies involving human participants were in accordance with the ethical standards of the University of Toronto Ethics Board per Protocol \#: 00038186 .

\section{References}

[1] R. J. Beichner and J. Saul, "Student-centered activities for large- enrollment university physics (SCALE- UP)," in Minneapolis, MN, 1999, pp. 43-52.

[2] T. Birdwell et al, "Active Learning Classroom Observation Tool: A Practical Tool for Classroom Observation and Instructor Reflection in Active Learning Classrooms," Journal on Centers for Teaching and Learning, vol. 8, pp. 28-50, 2016.

[3] B. M. Capobianco, J. DeLisi and J. Radloff, "Characterizing elementary teachers' enactment of highleverage practices through engineering design-based science instruction," Science Education (Salem, Mass.), vol. 102, (2), pp. 342-376, 2018. Available: https://onlinelibrary.wiley.com/doi/abs/10.100 2/sce.21325. DOI: $10.1002 /$ sce.21325.

[4] M. T. Chi and R. Wylie, "The ICAP framework: Linking cognitive engagement to active learning outcomes," Educational Psychologist, vol. 49, (4), pp. 219-243, 2014.

[5] D. K. Cohen and D. L. Ball, "Instruction, capacity, and improvement. CPRE research report series RR-43." Consortium for Policy Research in Education, June. 1999.

[6] M. Cole and Y. Engeström, "A cultural-historical approach to distributed cognition," .

[7] P. Dillenbourg, "Design for classroom orchestration," Computers and Education, vol. 69, pp. 485-492, 2013.

Available: http://dx.doi.org/10.1016/j.compedu.2013.04. 013. DOI: 10.1016/j.compedu.2013.04.013.

[8] Y. J. Dori and J. Belcher, "How Does TechnologyEnabled Active Learning Affect Undergraduate Students' Understanding of Electromagnetism Concepts?" The Journal of the Learning Sciences, vol. 14, (2), pp. 243279, 2005.

Available: http://www.tandfonline.com/doi/abs/10.1207/ s15327809j1s1402 3. DOI: 10.1207/s15327809j1s1402_3. 
[9] E. Dringenberg and R. E. H. Wertz, "AC 2012-3324: DEVELOPMENT OF THE SCIENCE AND ENGINEER- ING CLASSROOM LEARNING OBSERVATION PROTOCOL," .

[10] S. Freeman et al, "Active learning increases student performance in science, engineering, and mathematics," Proc. Natl. Acad. Sci. USA, vol. 111, (23), pp. 8410-8415, 2014.

Available: http://www.pnas.org/content/111/23/8410.abst ract. DOI: 10.1073/pnas.1319030111.

[11] G. Gay and H. Hembrooke, Activity-Centered Design: An Ecological Approach to Designing Smart Tools and Usable Systems. Cambridge, MA: MIT Press, 2004.

[12] U. Hedestig and V. Kaptelinin, "Re-contextualization of teaching in a videoconference-based environment," in 2002, pp. 179-188.

[13] U. Hedestig and V. Kaptelinin, "Facilitator's Roles in a Videoconference Learning Environment," Inf Syst Front, vol. 7, (1), pp. 71-83, 2005. DOI: 10.1007/s10796-005-5339-6.

[14] C. Henderson and M. H. Dancy, "Physics faculty and educational researchers: Divergent expectations as barriers to the diffusion of innovations," American Journal of Physic, vol. 76, (1), pp. 79-91, 2008.

[15] M. Hora and J. Ferrare, "The Teaching Dimensions Observation Protocol (TDOP)

2.0," pp. 20, 2014.

[16] M. T. Hora, "Toward a Descriptive Science of Teaching: How the TDOP Illuminates the Multidimensional Nature of Active Learning in Postsecondary Classrooms," Science Education, vol. 99, (5), pp. 783-818, 2015.

[17] M. T. Hora and J. J. Ferrare, "Instructional Systems of Practice: A Multidimensional Analysis of Math and Science Undergraduate Course Planning and Classroom Teaching," The Journal of the Learning Sciences, vol. 22, (2), pp. 212-257, 2013.

Available: http://www.tandfonline.com/doi/abs/10.1080/ 10508406.2012.729767. DOI:

10.1080/10508406.2012.729767.

[18] M. Hora and J. Ferrare, "A Review of Classroom Observation Techniques in Postsecondary Settings," WCER Working Paper no. 2013-1, 2013.

[19] D. H. Jonassen, "Revisiting activity theory as framework for designing student-centered learning environments," in Theoretical Foundations of Learning Environments, 1st ed., D. H. Jonassen and S. M. Land, Eds. Lawrence Erlbaum Associates, Inc., 2000, pp. 89122.
[20] D. H. Jonassen and L. Rohrer-Murphy, "Activity Theory as a Framework for Designing Constructivist Learning Environments," Etr\&d, vol. 47, (1), pp. 61-79, 1999. Available: https://www.jstor.org/stable/30220230. DOI: $10.1007 / \mathrm{BF} 02299477$.

[21] V. Kaptelinin and B. A. Nardi, Acting with Technology: Activity Theory and Interaction Design. Cambridge, Mass.: MIT Press, 2006.

[22] P. Kranzfelder et al, "The Classroom Discourse Observation Protocol (CDOP): A quantitative method for characterizing teacher discourse moves in undergraduate STEM learning environments," PloS One, vol. 14, (7), 2019. Available: https://doi.org/10.1371/journal.pone.0219019. DOI: 10.1371/journal.pone.0219019.

[23] M. Menekse et al, "Differentiated Overt Learning Activities for Effective Instruction in Engineering Classrooms," Journal of Engineering Education (Washington, D.C.), vol. 102, (3), pp. 346-374, 2013. Available: https://onlinelibrary.wiley.com/doi/abs/10.100 2/jee.20021. DOI: 10.1002/jee.20021.

[24] E. Scanlon and K. Issroff, "Activity Theory and Higher Education: evaluating learning technologies," Journal of Computer Assisted Learning, vol. 21, (6), pp. 430-439, 2005. . DOI: 10.1111/j.1365-2729.2005.00153.x.

[25] M. K. Smith et al, "The Classroom Observation Protocol for Undergraduate STEM (COPUS): A New Instrument to Characterize University STEM Classroom Practices," CBE Life Sci Educ., vol. 12, (4), pp. 618-627, 2013.

Available: https://www.ncbi.nlm.nih.gov/pmc/articles/P MC3846513/.

[26] R. Talbert and A. Mor-Avi, A Space for Learning: $A$ Review of Research on Active Learning Spaces. 2018.

[27] A. Van Beek and S. McCahan, Review of In-Class Active Learning Observation Protocols, Accepted, Proceedings of the ASEE Annual Meeting, 2021.

[28] S. Van Horne et al, "Promoting Active Learning in Technology-Infused TILE Classrooms at the University of Iowa," Journal of Learning Spaces, vol. 1, (2), 2012. Available: https://doaj.org/article/22c4a3dde9ea4a11aaa8 2abe2865244d.

[29] J. M. Wilson and W. C. Jennings, "Studio courses: How information technology is changing the way we teach, on campus and off," Jproc, vol. 88, (1), pp. 72-80, 2000. Available: https://ieeexplore.ieee.org/document/811603. DOI: $10.1109 / 5.811603$. 


\section{Appendix A: TDOP+ \\ (adapted from TDOP) [15]}

The following list of codes shows the existing TDOP dimensions as well as the modifications proposed in this paper. A more thorough description of each code can be found in [15]. The additions are added to the end of each dimension and are bolded. Modifications were made based on dimensions included in the ALCOT [2] as well as observations and room-specific technologies and tools.

The TDOP Dimensions are:

1. Teaching methods
a. Teacher-focused instruction
b. Student-focused instruction

2. Student-teacher dialogue
a. Teacher-led dialogue
b. Student-led dialogue

3. Pedagogical Strategies

4. Instructional Technology

5. Potential Student Cognitive Engagement (No Additions)

6. Student Engagement (No Additions)

\section{Teaching Methods}

Teacher-focused instruction

\begin{tabular}{|c|c|c|}
\hline \multirow[t]{10}{*}{ Existing: } & $\mathrm{L}$ & Lecturing \\
\hline & LW & Lecturing while writing \\
\hline & LVIS & Lecturing from pre-made visuals \\
\hline & SOC-L & Socratic lecture \\
\hline & LDEM & $\begin{array}{l}\text { Lecturing with demonstration of } \\
\text { phenomena }\end{array}$ \\
\hline & WP & Working out problems \\
\hline & IND & Individualized instruction \\
\hline & MM & Multimedia \\
\hline & A & Assessment \\
\hline & AT & Administrative task \\
\hline \multirow[t]{3}{*}{ Added: } & $\mathbf{P}$ & $\begin{array}{l}\text { Preparation/Planning } \\
\text { (Added from } A L C O T \text { ) }\end{array}$ \\
\hline & ORG & $\begin{array}{l}\text { Organization } \\
\text { (Added from } A L C O T)\end{array}$ \\
\hline & $\mathbf{R E}$ & $\begin{array}{l}\text { Review/Recap Summary } \\
\text { (Added from ALCOT) }\end{array}$ \\
\hline
\end{tabular}

Student-focused instruction

$\begin{array}{lll}\text { Existing: } & \text { SGW } & \text { Small group work/discussion } \\ & \text { DW } & \text { Deskwork } \\ \text { SP } & \text { Student presentation }\end{array}$

2. Student-Teacher Dialogue

Teacher-led dialogue

Existing: IRQ Instructor rhetorical question

IDQ Instructor display question

\begin{tabular}{|c|c|c|}
\hline & ICQ & Instructor comprehension question \\
\hline Added: & CS & $\begin{array}{l}\text { Instructor-Student Content } \\
\text { Sharing (Added from Observation) }\end{array}$ \\
\hline \multicolumn{3}{|c|}{ Student-led dialogue } \\
\hline & SQ & Student question \\
\hline & SR & Student response to teacher questior \\
\hline & PI & Peer interactions \\
\hline
\end{tabular}

3. Pedagogical Strategies

$\begin{array}{lll}\text { Existing: } & \text { HUM } & \text { Humor } \\ & \text { ANEX } & \text { Anecdote/example } \\ & \text { ORG } & \text { Organization } \\ \text { Added: } & \text { MEP } & \begin{array}{l}\text { Emphasis } \\ \text { Managing Expectations (Added } \\ \text { from ALCOT) } \\ \text { Collaborative/Complementary } \\ \text { Teaching } \\ \text { (Added from Observation) } \\ \text { Regrouping (Added from } \\ \text { Observation) }\end{array} \\ \text { CT } & \text { RG } & \end{array}$

\section{Instructional Technology}

\begin{tabular}{|c|c|c|}
\hline Existing: & $\mathrm{CB}$ & Chalkboard \\
\hline & WB & Whiteboard \\
\hline & $\mathrm{OP}$ & Overhead projector/transparencies \\
\hline & PP & PowerPoint or other digital slides \\
\hline & CRT & Clicker response systems \\
\hline & $\mathrm{D}$ & Demonstration Equipment \\
\hline & DT & Digital tablet \\
\hline & M & Movie, documentary, video clips, etc. \\
\hline & SI & Simulation \\
\hline & WEB & Website \\
\hline & M & $\begin{array}{l}\text { Movie, documentary, video clips, or } \\
\text { Youtube video }\end{array}$ \\
\hline Addekdd & PFom & $\begin{array}{l}\text { Coqument Camera (Added based on } \\
\text { Room Equipment) }\end{array}$ \\
\hline & CAM & $\begin{array}{l}\text { Camera (Added based on Room } \\
\text { Equipment) }\end{array}$ \\
\hline & MIC & $\begin{array}{l}\text { Student Microphone use (Added } \\
\text { based on Room Equipment) }\end{array}$ \\
\hline & SD & $\begin{array}{l}\text { Student Display Use (Added based } \\
\text { on Room Equipment) }\end{array}$ \\
\hline & TECH & $\begin{array}{l}\text { Issue requiring technical assistance } \\
\text { (Added from Observation) }\end{array}$ \\
\hline & TI & $\begin{array}{l}\text { Timer (Added based on Room } \\
\text { Equipment) }\end{array}$ \\
\hline & WC & $\begin{array}{l}\text { Wireless Input Connection (Added } \\
\text { based on Room Equipment) }\end{array}$ \\
\hline
\end{tabular}


WIFI Wifi Use (Added based on Room Equipment)

STI Student Table Input (Added based on Room Equipment)

\section{Potential Student Cognitive Engagement}

$\begin{array}{ll}\text { CNL } & \text { Making connections to own } \\ & \text { lives/specific cases } \\ \text { PS } & \text { Problem solving } \\ \text { CR } & \text { Creating }\end{array}$

\section{Student Engagement}

$\begin{array}{ll}\text { VHI } & \text { Very High Volume } \\ \text { HI } & \text { High Volume } \\ \text { MED } & \text { Medium Volume } \\ \text { LO } & \text { Low Volume }\end{array}$

